This study was supported by a grant from the U.S. Atomic Energy Commission.

\section{WiLliam CoHen}

Department of Organic Chemistry and Enzymology,

\section{Fordham University,} New York 58. June 30.

${ }^{1}$ Winnick, T., Cone, W. H., and Greenberg, D. M., J. Biol. Chem.,
153,465 (1944). 2 Bernhard, S. A., and Gutfreund, H., Biochem. J., 63, 61 (1956).

${ }^{3}$ Olcott, H. S., and FraenkeI-Conrat, H., Chem. Rev., 41, 151 (1947).

${ }^{4}$ Cohen, Wm., Bier, M., and Nord, F. F., Arch. Biochem. Biophys., 67, 479 (1957).

${ }^{5}$ Cohen, Wm., Bier, M., and Nord, F. F., Areh. Biochem. Biophys., 76, 204 (1958).

${ }^{6}$ Terminiello, L., Sri Ram, J., Bier, M., and Nord, F. F., Arch. Biochem. Biophys., 5", 252 (1955).

\section{Separation of Chalcones, Flavanones and Flavonols by Chromatography with Polyamide}

Polyamide had been used as an adsorbent in chromatography by Carelli, Liquori and $\mathrm{Mele}^{\mathrm{x}}$, as well as by Grassmann, Hoermann and Hartl ${ }^{2}$, for the separation of phenols. Recently, Hoerhammer, Wagner and Leeb ${ }^{3}$ used it for the separation of flavones and their glycosides, and for the quantitative removal of tanning substances from plant extracts containing flavones.

The complete conversion of chalcones into flavanones is very unusual, and separation by recrystallization is often difficult. It is possible, however, to fractionate chalcones and flavones on a column with powdered polyamide; the chalcone is retained in the upper part of the column, whereas the flavanone is readily eluted with water, aqueous alcohol or aqueous acetone respectively. By this means the simple chalcones can be separated from the corresponding isomeric flavanones; for example, 2hydroxy-, 4'-hydroxy-, 4'-methoxy-, 3'-methoxy-4'hydroxy-flavanones. The chalcone that remains in the upper part of the column can be eluted with absolute methanol. If plant extracts are used that contain both chalcones and flavanones, for example butin and butein, the best separation is obtained by aqueous alcohol mixtures. The plant extracts are either dried on, or triturated with, polyamide and the combination placed on a polyamide column. The flavanone is first eluted with mixtures of water and alcohols or ketones, and then the chalcone with the pure solvent. The concentration of the solvent mixtures used for the separation depends on the substance, and is found by the usual alteration of the elution medium in sorption chromatography.

It is often not easy to isolate pure flavanoids, as distinct from the fraction containing these substances, and the process often involves considerable losses of substance ${ }^{4}$. Chromatographic procedures are especially important in this connexion but all the usual substances known as adsorbents cannot be used for the separation of flavonols, especially those that adsorb these compounds irreversibly. Polyamide is an adsorbent that has not hitherto been examined in detail in this respect.

In further work on the hydroxy phenyl-benzo- $\gamma$ pyrones it was found that separation of flavonol and flavanonol was possible on a column of polyamide. For these investigations a crude sample of dihydroquercetin that contained a small amount of quercetin was used. The extract obtained from the crude flavanonol by means of diethyl ether was dried on powdered polyamide, and then put on a column containing the same adsorbent made up in water. By elution with aqueous ethanol (20-30 per cent of ethanol) the dihydroquercetin was first obtained and then, by elution with pure ethanol, the quercetin. Both of them were paper-chromatographically pure.

I wish to thank Dr. D. W. Glennie, of the Oregon Forest Research Center, Cornwallis, for the dihydroquercetin.

\section{Richard NeU}

Messrs. Dr. Willmar Schwabe, Ltd.,

Chemical Research Laboratories, Karlsruhe Durlach.

$$
\text { June } 9 .
$$

1 Carelli, B., Liquori, A. M., and Mele, A., Nature, 176, 70 (1955).

${ }^{2}$ Grassmann, W., Hoermann, H., and Hart1, A., Makromolekulare Chemie, 21,37 (1956).

${ }^{3}$ Hoerhammer, L., Wagner, H., and Leeb, W., Naturwiss., 44, 513 (1957).

Kurth, E. F., Hegert, H. L., and Ross, J. D., J. Amer. Chem. Soc. ry, 1621 (1955).

\section{Reactivity of Sulphonyloxyl Groups with Halides in the Carbohydrate Series}

IT has recently been shown ${ }^{1}$ that refluxing 6-O-mesyl-1 : 2-3 : 4-di-O-isopropylidene-D-galactopyranose $^{2}$ (i) and 3 : 5-O-benzylidene-6-O-mesyl-1 : 2O-isopropylidene-D-glucofuranose ${ }^{3}$ (ii) with potassium fluoride in ethanediol, yields the 6-deoxy-6-fluoro-Dgalactopyranose and $D$-glucofuranose derivatives, respectively. A notable difference in the reactivity of the two sugars towards potassium fluoride was found (Table 1).

\begin{tabular}{|c|c|c|c|}
\hline Compound & Temperature & $\begin{array}{l}\text { Time } \\
\text { (min.) }\end{array}$ & $\begin{array}{l}\text { Per cent } \\
\text { yield of } \\
\text { fluorosugar }\end{array}$ \\
\hline $\begin{array}{l}\text { 6-O-Mesyl*-D-galactose } \\
\text { derivative (i) }\end{array}$ & 200 & 75 & 60 \\
\hline $\begin{array}{l}\text { 6-O-Mesyl-D-glucose } \\
\text { derivative (ii) }\end{array}$ & 200 & $2 \cdot 5$ & 75 \\
\hline
\end{tabular}

A similar qualitative difference in the reactivity of terminal sulphonyloxyl groups of the above D-galactose and D-glucose derivatives has also been observed in the case of iodine exchange reactions, using sodium iodide in acetone (review by Tipson'). It is to be expected that the difference in size (and electronic properties) between the iodide and fluoricle ions will be one of the many factors which will influence the reactivity of the sulphonyloxyl groups. These results with fluorine and iodine exchange reactions, however, suggest the importance of the actual stereochemistry of the sugar molecule in exchange reactions of this type.

Structures (I) and (II), representing the D-galactopyranes (i) and D-glucofuranose (ii) derivatives, respectively, in their most stable cis-anti-cis (rings $A B C)$ steric arrangement, are written in accordance with the convention suggested by Mills ${ }^{5} a$. It is significant that if ring $B$ in (I) and ring $A$ in (II) retain their most stable $C 1$ chair conformation, then the residue $-\mathrm{CH}_{2} \mathrm{OMs}$ is equatorially disposed in (I) and axial in (II). 